\title{
Study on numerical well test for discrete-fracture cavity model
}

\author{
Fangfang Chen"1, a, Shaoji Hou 2, 3, b*, Zhiwen Ding ${ }^{1, \text { c }}$, Yuewu Liu², 3, d, \\ Jianglong Xue ${ }^{1, e}$ and Qi Li ${ }^{2, f}$
}

${ }^{1}$ Research Institute of Exploration and Development, Tarim Oilfield Company, PetroChina, Korla 841000, China

${ }^{2}$ Institute of Mechanics, Chinese Academy of Sciences, Beijing, 100190 China

${ }^{3}$ University of Chinese Academy of Sciences, Beijing, 100149 China

achenff-tlm@petrochina.com.cn, bhoushaoji@imech.ac.cn, cdingzw-tlm@petrochina.com.cn, diluyuewulxs@126.com, exuejl-tlm@petrochina.com.cn, fliqi0620@163.com

Keywords: Discrete-fracture model, Finite element method, Numerical well test, Vuggy carbonate reservoir.

\begin{abstract}
For the fractured vuggy carbonate reservoir, the existing well test models are difficult to apply because of many factors, such as the rock structure, the structural fracture and the dissolution. To solve this problem and represent the complicated flow in carbonate reservoir, we propose a finite element numerical well test model to analyze the fractured carbonate reservoirs, which are descripted from different regional reservoir flow conditions and the fluid characteristics in the numerical model. The numerical model of fractured vuggy carbonate reservoir can be used to simulate the process of pressure change, more accurate flow details of fracture cave type carbonate reservoir are described, and theoretical guidance for the development of fractured vuggy carbonate reservoirs are provided.
\end{abstract}

\section{Introduction}

With the global energy shortage, geographical control and the world's oil and gas exploration and development of the degree of gradual deepening. In recent years, many unconventional reservoirs and complex reservoir to begin development. Vuggy carbonate reservoirs are one of the most important categories. Because the reservoirs of the fractured vuggy reservoir are characterized by heterogeneity, complex spatial structure, random distribution of cavities, different size of caves and large inclination of cracks, the development of fractured vuggy reservoirs are still in the exploratory stage in the world [1-3]. Compared with the conventional reservoir, the spatial dispersion is the prominent characteristic of the fractured vuggy reservoir [4]. At the same time, the spatial distribution of the reservoir has a strong randomness, which is difficult to solve the geological modeling, reservoir numerical simulation and numerical well test [5-6]. The existing numerical models of fractured vuggy reservoirs include multiple medium model [7-8], coupled flow model [9], discrete medium well test model [10-11] and so on. The multiple medium model is to consider the cave as continuous medium, but in fact the cave is spatially dispersed, the representative elementary volume(REV) does not exist, so it is equivalent to continuous medium is not suitable.. The coupling flow model takes into account the discrete characteristics of the fractured media, but the fluid flow in the karst cave is described by the $\mathrm{N}-\mathrm{S}$ equation leading to computational cost is too high, which is difficult to be used in numerical well test analysis.

Based on the discrete and random distribution of fracture cavity in fractured vuggy reservoir and the seismic and geological data and the discrete crack model, the numerical simulation model of discrete fracture cavity is established by considering the statistical characteristics of the physical parameters of karst cave and fracture. To solve the mathematical model, the Galerkin Finite Elements Method was used together. The influence of the factors such as the distribution of fracture and hole, wellbore storage and skin effect on the well test curve is analyzed. The well test curve of the two 
dimensional discrete well test model is obtained, and the characteristic curves of the bottom hole pressure response are analyzed.

\section{Numerical Well Test Model based on FEM}

The finite element numerical method is introduced into the well test, and the reservoir is described by the discretization variable. The conclusion is more accurate and the conclusion is more in line with the actual characteristics of the reservoir. The finite element numerical well test analysis method starts with the establishment of the basic physical model. For a particular reservoir, it is usually composed of 4 basic models: internal boundary model, reservoir model, outer boundary model and fluid model.

\subsection{Physics Model}

According to the test curve and the corresponding geological characteristics, two types of well models are established based on seismic reflection data: (1)WIC-Well Inside of the Cave Model, (2) WOC-Well Outside of the Cave. As shown in Fig 1.

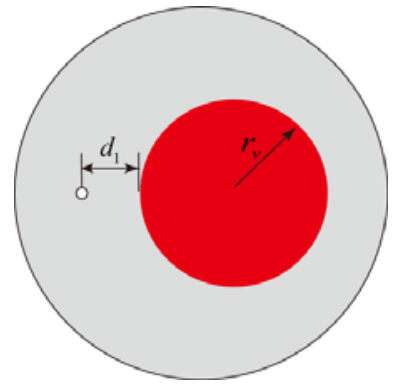

(a) WOC model

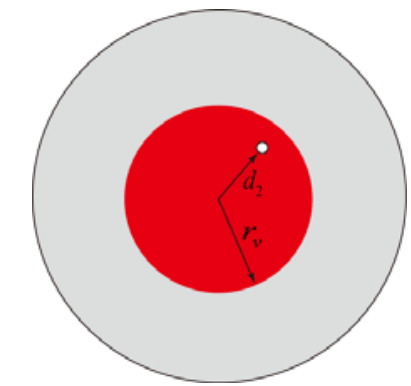

(b) WIC model

Fig. 1 Two-dimensional discrete fracture discrete-fracture cavity numerical well test model

$d_{1}$ is the dimensionless distance from the wellbore to the cave, $r_{\mathrm{v}}$ is the radius of the cave, and $d_{2}$ is the distance from the center of the cave to the well.

In order to establish the model of well testing in the fractured vuggy carbonate reservoir. The following assumptions are considered:

Seepage of a single phase weakly compressible fluid in a horizontal, equal thickness, top closed layer;

In the stratum, the fracture cavity is developed, the well is drilled or not drilled, and the well is produced with constant output;

The seepage in the fracture cavity and other areas of the formation satisfies Darcy's law;

The wellbore and skin effects are considered, the effects of gravity and capillary pressure are neglected, the temperature is constant, Other physical and chemical effects are not considered.

\subsection{Mathematics Model}

All manuscripts must be in English, also the table and figure texts, otherwise we cannot publish your paper. Please keep a second copy of your manuscript in your office. When receiving the paper, we assume that the corresponding authors grant us the copyright to use the paper for the book or journal in question. Should authors use tables or figures from other Publications, they must ask the corresponding publishers to grant them the right to publish this material in their paper.

The karst caves can be represented by a region with different permeability and porosity, and the permeability and porosity of the karst caves and fractures are often large. The mathematical model can be expressed as:

Substrate region:

$$
\frac{\partial^{2} p_{1 \mathrm{D}}}{\partial x_{\mathrm{D}}^{2}}+\frac{\partial p_{1 \mathrm{D}}}{\partial y_{\mathrm{D}}^{2}}=\frac{1}{C_{\mathrm{D}} \mathrm{e}^{2 S}} \frac{\partial p_{1 \mathrm{D}}}{\partial T_{\mathrm{D}}}
$$

Fracture cavity region:

$$
\frac{\partial^{2} p_{2 \mathrm{D}}}{\partial x_{\mathrm{D}}^{2}}+\frac{\partial p_{2 \mathrm{D}}}{\partial y_{\mathrm{D}}^{2}}=\frac{1}{C_{\mathrm{D}} \mathrm{e}^{2 S}} \frac{\omega}{M} \frac{\partial p_{2 \mathrm{D}}}{\partial T_{\mathrm{D}}}
$$


Initial condition:

$$
p_{1 \mathrm{D}}\left(x_{D}, y_{D}, 0\right)=0, p_{2 \mathrm{D}}\left(x_{D}, y_{D}, 0\right)=0
$$

Wellbore boundary condition:

$$
\left.\sum_{j=1}^{N} L_{j} \frac{\partial p_{\mathrm{D}}}{\partial \mathrm{n}}\right|_{\Gamma_{\mathrm{i}}}=2 \pi\left(-1+\frac{d p_{\mathrm{wD}}}{d T_{\mathrm{D}}}\right)
$$

Interface condition of karst and matrix:

$$
\begin{aligned}
& \left.p_{1 \mathrm{D}}\left(T_{\mathrm{D}}\right)\right|_{\Gamma_{\mathrm{v}}}=\left.p_{2 \mathrm{D}}\left(T_{\mathrm{D}}\right)\right|_{\Gamma_{\mathrm{v}}} \\
& \left.\frac{\partial p_{1 \mathrm{D}}}{\partial n_{\mathrm{v}}}\right|_{\Gamma_{\mathrm{v}}}=\left.M \frac{\partial p_{2 \mathrm{D}}}{\partial n_{\mathrm{v}}}\right|_{\Gamma_{\mathrm{v}}}
\end{aligned}
$$

Reservoir boundary condition:

Sealing boundary conditions:

$$
\left.\frac{\partial p_{\mathrm{D}}}{\partial \mathrm{n}}\right|_{\Gamma_{\mathrm{e}}}=0
$$

Constant-pressure boundary condition:

$$
\left.p_{\mathrm{D}}\right|_{\Gamma_{\mathrm{e}}}=0
$$

Where: $p_{1 \mathrm{D}}$ is dimensionless pressure for fracture systems; $p_{2 \mathrm{D}}$ is dimensionless pressure of cavern system; $p_{\mathrm{wD}}$ is dimensionless pressure for bottomhole; $T_{\mathrm{D}}$ is dimensionless time; $C_{\mathrm{D}}$ is dimensionless wellbore storage coefficient; $x_{\mathrm{D}}$ and $y_{\mathrm{D}}$ is dimensionless coordinate distance; $S$ is skin factor; $\omega$ is fracture energy storage ratio; $M$ is mobility ratio of fracture cavity.

\subsection{Discrete Solution of Mathematical Model}

\subsubsection{Establishment of Finite Element Mesh}

The finite element numerical well test analysis method is applied for the discretization of fractured vuggy carbonate reservoir selection triangular mesh. The grid around the wellbore and around the cavity is densified. For reservoir wells, different seepage between the units and the radial boundary interference model and interference model, angle model and model fragments and other methods combined, and ultimately the formation of a reservoir can accurately reflect the geological characteristics of the composite element mesh model. As shown in Fig 2.
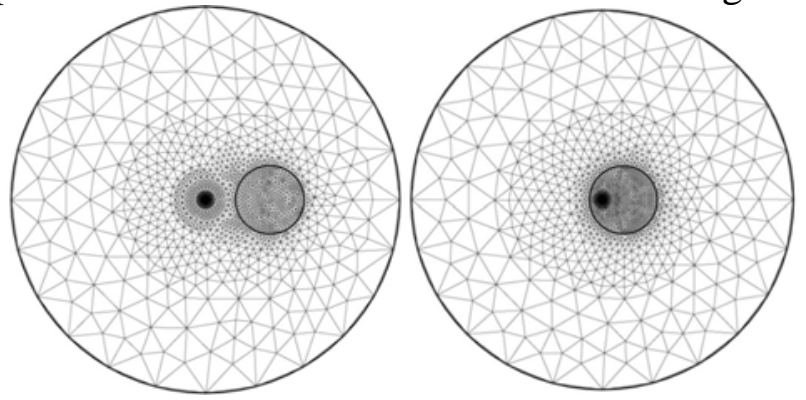

(a) Well Outside of the Cave (b) Well Inside of the Cave

Fig. 2 The grid of WOC model and WIC model

\subsubsection{Establishment of Finite Element Mesh}

The mathematical model is discretized in time and space and using the Galerkin weighted residual method, the triangle is chosen as the basic unit, and the weight function is the interpolation function. The discrete element finite element equation of the matrix region can be expressed as: 


$$
\begin{aligned}
& A\left(b_{i}^{2}+c_{i}^{2}+\frac{1}{6 C_{\mathrm{D}} \mathrm{e}^{2 S} \Delta T}\right) p_{1 i}^{\mathrm{e}, n+1}+A\left(b_{i} b_{j}+c_{i} c_{j}+\frac{1}{12 C_{\mathrm{D}} \mathrm{e}^{2 S} \Delta T}\right) p_{1 j}^{\mathrm{e}, n+1}+ \\
& A\left(b_{i} b_{k}+c_{i} c_{k}+\frac{1}{12 C_{\mathrm{D}} \mathrm{e}^{2 S} \Delta T}\right) p_{1 k}^{\mathrm{e}, n+1}-\frac{L}{3} \frac{\partial p_{1 i}^{\mathrm{e}, n+1}}{\partial \mathrm{n}}-\frac{L}{6} \frac{\partial p_{1(j, k)}^{\mathrm{e}, n+1}}{\partial \mathrm{n}}= \\
& \frac{A}{6 C_{\mathrm{D}} \mathrm{e}^{2 S} \Delta T} p_{1 i}^{\mathrm{e}, n}+\frac{A}{12 C_{\mathrm{D}} \mathrm{e}^{2 S} \Delta T} p_{1 j}^{\mathrm{e}, n}+\frac{A}{12 C_{\mathrm{D}} \mathrm{e}^{2 S} \Delta T} p_{1 k}^{\mathrm{e}, n}
\end{aligned}
$$

The finite element equation of karst area:

$$
\begin{aligned}
& A M\left(b_{i}^{2}+c_{i}^{2}+\frac{1}{6 C_{\mathrm{D}} \mathrm{e}^{2 S} \Delta T}\right) p_{2 i}^{\mathrm{e}, n+1}+A M\left(b_{i} b_{j}+c_{i} C_{j}+\frac{1}{12 C_{\mathrm{D}} \mathrm{e}^{2 S} \Delta T}\right) p_{2 j}^{\mathrm{e}, n+1}+ \\
& A M\left(b_{i} b_{k}+c_{i} C_{k}+\frac{1}{12 C_{\mathrm{D}} \mathrm{e}^{2 S} \Delta T}\right) p_{2 k}^{\mathrm{e}, n+1}-\frac{L M}{3} \frac{\partial p_{2 i}^{\mathrm{e}, n+1}}{\partial \mathrm{n}}-\frac{L M}{6} \frac{\partial p_{2(j, k)}^{\mathrm{e}, n+1}}{\partial \mathrm{n}}= \\
& \frac{A M}{6 C_{\mathrm{D}} \mathrm{e}^{2 S} \Delta T} p_{2 i}^{\mathrm{e}, n}+\frac{A M}{12 C_{\mathrm{D}} \mathrm{e}^{2 S} \Delta T} p_{2 j}^{\mathrm{e}, n}+\frac{A M}{12 C_{\mathrm{D}} \mathrm{e}^{2 S} \Delta T} p_{2 k}^{\mathrm{e}, n}
\end{aligned}
$$

The total flow of wellbore boundary is equal to the total production:

$$
\left.\sum_{j=1}^{N} L_{j} \frac{\partial p_{\mathrm{D} j}^{e, n+1}}{\partial \mathrm{n}}\right|_{\Gamma_{i}}-\frac{1}{2 \pi} \frac{p_{\mathrm{wD}}^{\mathrm{e}, n+1}}{\Delta T}=\frac{1}{2 \pi}\left(-1-\frac{p_{\mathrm{wD}}^{\mathrm{e}, n}}{\Delta T}\right)
$$

The pressure of each point on the wellbore boundary is equal to the bottom hole pressure:

$$
\left.p_{\mathrm{Dj}}\right|_{\Gamma_{\mathrm{i}}}=p_{\mathrm{wD}}
$$

Simultaneous equations (10), (11), (12), the element stiffness equation is assembled into the global stiffness equation, and the pressure values at each node can be obtained.

\section{Model Calculation Results and Analysis}

The model is used to analyze the field data. The well A in the Tarim Oilfield is located in the Ordovician, which belongs to a typical fractured vuggy carbonate reservoir. The physical parameters of wells and reservoirs: porosity is $4.2 \%$, the effective thickness of reservoir is $67 \mathrm{~m}$, the well bore radius is $0.079 \mathrm{~m}$, the compression coefficient is $0.00114 \mathrm{MPa}^{-1}$, the formation oil volume factor is $1.12 \mathrm{~m}^{3} / \mathrm{m}^{3}$, the viscosity of crude oil is $1.49 \mathrm{mPa} \bullet$ s. The wells in the drilling process is not leaking venting phenomenon, we select the WOC model to explain the well. The results are interpreted by means of typical well test curve: the well storage coefficient is $0.291 \mathrm{~m}^{3} / \mathrm{MPa}$, the skin factor is -4.98 , the permeability of cave near well is $8278 \mathrm{mD}$, the karst cave radius is $185 \mathrm{~m}$, the nearest distance between well and cave is $35 \mathrm{~m}$. As shown in Fig 3, a double logarithmic curve fitting.

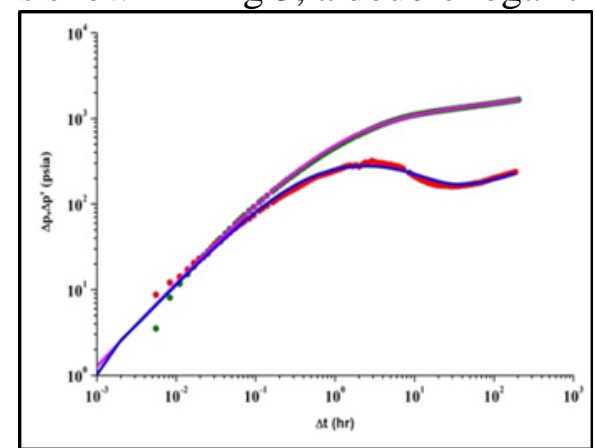

Fig. 3 Well A double logarithmic curve fitting

The well B in the Tarim Oilfield is located in the Ordovician, which also belongs to a typical fractured vuggy carbonate reservoir. The physical parameters of wells and reservoirs: porosity is $4.02 \%$, the effective thickness of reservoir is $43 \mathrm{~m}$, the well bore radius is $0.0762 \mathrm{~m}$, the compression coefficient is $0.00114 \mathrm{MPa}^{-1}$, the formation oil volume factor is $1.037 \mathrm{~m}^{3} / \mathrm{m}^{3}$, the viscosity of crude oil 
is $2.19 \mathrm{mPa} \bullet \mathrm{s}$. In the process of drilling, the drilling fluid $640.92 \mathrm{~m}^{3}$ was lost, and empting drilling tool $4.6 \mathrm{~m}$, which indicates that the well is drilled with a large fracture cavity. We select the WIC model to explain the well. The results are interpreted by means of typical well test curve: the well storage coefficient is $13.6 \mathrm{~m}^{3} / \mathrm{MPa}$, the skin factor is -4.46 , the permeability of cave near well is $3404 \mathrm{mD}$, the karst cave radius is $112 \mathrm{~m}$, the nearest distance between well and cave is $45 \mathrm{~m}$. As shown in Fig 4, a double logarithmic curve fitting.

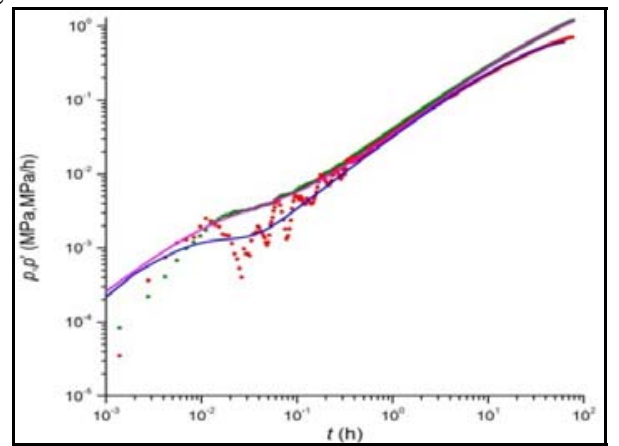

Fig. 4 Well B double logarithmic curve fitting

\section{Conclusion}

The finite element numerical well test analysis method is used to describe and solve the two-dimensional discrete-fracture cavity model, the results are as follows:

(1) In this paper, a two-dimensional discrete-fracture cavity model is established. The model considers two types: WIC-Well Inside of the Cave Model, WOC-Well Outside of the Cave. The results showed: (1)the WIC model and radial composite model are similar, but can be considered well and the fracture cavity is not concentric, the larger the eccentricity distance, the larger the amplitude of the first radial flow section of the bottom hole pressure response; (2)The typical characteristic of the bottom hole pressure response curve of the WOC model which a concave section on the pressure derivative, which is very similar to the double medium model.

(2) Based on the mathematical model of numerical well testing of discrete-fracture cavity model and combining with the actual production data and test data, the application of two well in Tarim Oilfield is analyzed. Finally, the established mathematical model can well explain the process of pressure change in the production process, and has a good consistency with the actual production.

\section{Acknowledgments}

This work was financially supported by the National Science and Technology Major Projects (Grant no. 2011ZX05004-004).

This work was financially supported by China Postdoctoral Science Fund Project (2016M601142).

\section{References}

[1]. Zheng S Q, Li Y, Zhang H F. Fracture-cavity network model for fracture-cavity carbonate reservoir [J]. Zhongguo Shiyou Daxue Xuebao, 2010, 34(3):72-75+79.

[2]. Jia H, Pu W, Liao R, et al. Vuggy-Fractured Carbonate Reservoirs Physical Simulation Methods [J]. Petroleum Drilling Techniques, 2010, 38(6):102-108.

[3]. Li W, Hou J, Ding G, et al. Remaining oil types and influence factors for fractured-vuggy carbonate reservoir [J]. Fault-Block Oil \& Gas Field, 2013, 20(4):458-461.

[4]. Uraiet A, Raghavan R, Thomas G W. Determination of the orientation of a vertical fracture by interference tests [J]. Journal of Petroleum Technology, 1977, 29(1): 73-80.

[5]. Kutasov I M, Eppelbaum L V, Kagan M. Interference well testing-variable fluid flow rate [J]. Journal of Geophysics \& Engineering, 2007, 5(1): 86-91. 
[6]. Sheng J J. A new technique to determine horizontal and vertical permeability's from the time-delayed response of a vertical interference test [J]. Transport in Porous Media, 2009, 77(3): 507-527.

[7]. Tong D K, Liu W C, Xue L L. Flow Characteristics of Triple-permeability Model in Low Permeability Reservoir with Deformed Triple Porosity Medium [J]. Chinese Quarterly of Mechanics, 2010, 31(3):334-341.

[8]. Liu W C, Zhang S M, Liu X Y, et al. Dual-percolation model of low permeability reservoir with deformed triple media [J]. Special Oil \& Gas Reservoirs, 2010, 17(3):73-76.

[9]. Wen C Y, Zhi-Min D U, Tang S D, et al. A comparison of new fluid-solid coupling models for fracture-pore type carbonate gas reservoirs [J]. Natural Gas Industry, 2008, 28(8):89-91.

[10]. Wan Y, Liu Y. Three dimensional discrete-fracture-cavity numerical well test model for fractured-cavity reservoir [J]. Chinese Journal of Theoretical and Applied Mechanics, 2015, 47(6).

[11]. PENG Chao-yang, LONG Wu, DU Zhi-min, LI Chuan-liang. The numerical well test model of the discrete media network in fractured-vuggy carbonate reservoir [J]. Journal of Southwest Petroleum University, 32(6), 125-129. 